\title{
Étude sur l'anatomie de l'ovaire et du corps jaune de la chamelle
}

\author{
par M.A.F. TAYEB, M.V.Sc. (Le Caire) \\ Lecturer in Departement of Anatomy, Fouad I University, College of Veterinary
}

(Traduit par P.-C. BLIN)

Chef de Travaux, École vétérinaire Alfort

\section{INTRODUCTION}

L'étude de l'ovaire et du corps jaune de la chamelle est demeurée incomplète.

LESBRE (1903) décrit l'ovaire comme étant de la grosseur d'un pois ou d'une noisette. Il est dissimulé dans une sorte de cupule du ligament large.

Il rapporte également la présence de nombreux ovisacs à la surface de l'ovaire, qui lui donnent l'apparence d'une grappe de raisin. Le ligament rond est facile à mettre en évidence.

LEESE (1927) décrit l'ovaire comme un organe régèrement aplati mesurant environ $2 \mathrm{~cm}$. 5 de longueur.

ASDELL (1946) précise que la longueur du follicule de De Graaf est de 1 centimètre à $1 \mathrm{~cm}$. 5 environ et que, quand il est à maturité complète, elle atteint $2 \mathrm{~cm}$. 5 à 3 centimètres.

L'objet de cette étude est de décrire l'ovaire et le corps jaune de la chamelle macroscopiquement ef microscopiquement.

\section{MATÉRIEL D'ÉTUDE ET MÉTHODE}

Les ovaires ont été prélevés aux abattoirs du Caire et de Embaba. Les ovaires de 150 chamelles soudanaises gestantes et non-gestantes, de 3 à 5 ans d'âge, furent examinés d'abord in situ puis détachés des cadavres.

Par suite de la similitude du corps jaune de la chamelle avec une sphère comprimée latéralement, deux mensurations furent prises, une longitudinale et l'autre transversale, au lieu des trois 'mensurations de Hammond (1927) chez la vache. L'ovaire et le corps jaune ont été étudiés à la fois macroscopiquement et microscopiquement.

Nous n'avons trouvé aucune référence relative aux rapports existant entre la longueur, le poids, la différenciation embryologique et l'âge du fœius.

Dans ce travail, la longueur du foetus mesurée du vertex à la base de la queue a remplacé la considération de l'âge du foetus dans l'étude du développement de divers corps jaunes. La longueur moyenne du chamelon nouveau-né a été obtenue à partir de mensurations portant sur plusieurs sujets.

\section{L'OVATRE}

\section{Morphologie et position.}

L'ovaire se présente comme un organe lobulé, aplati, quelque peu rougeâtre, avec un contour circulaire. Les faces externe et interne sont légèrement convexes. Le bord libre inférieur convexe ne présente pas de cavité d'ovulation.

Le bord adhérent correspondant est presque rectiligne.

L'ovaire est enfermé dans un vaste repli conique ayant l'aspect d'une poche et dépcindant du mésosalpinx, connu chez d'autres espèces domestiques sous le nom de bourse ovarique. Le sommet de cette bourse, chez la chamelle, est en cul-de-sac et situé en dehors. L'extrémité interne correspondante de cette bourse forme un vaste orifice circulaire audessus et en dehors duquel pend sur le plancher de la bourse elle-même le morceau frangé.

Le ligament de l'ovaire se rattache à la face supérieure du ligament large près de son union avec le bord externe de la corne utérine. Le ligament de l'ovaire se présente sous forme d'un relief en cordon s'étendant de la face supérieure du ligament large au hile de l'ovaire correspondant, nettement délimité.

Son point d'origine se trouve à mi-distance entre le bord externe de la corne et l'ovaire lui-même.

In situ, l'ovaire se place au niveau de la $6^{\mathrm{e}}$ vertèbre lombaire et, même, entre celle-ci et la $7^{\mathrm{e}}$ lombaire à 6-7 centimètres de la crête iliaque.

Toutefois, cette position est variable et dépend de l'ampleur des viscères au voisinage.

L'ovaire se trouve à 45 centimètres des commissures vulvaires.

Chez l'animal adulte, l'ovaire pèse 2 à 4 grammes et mesure de 2 à 4 centimètres de long, 1,5 à 2 centimètres de large et 0,8 à 1 centimètre d'épaisseur. 


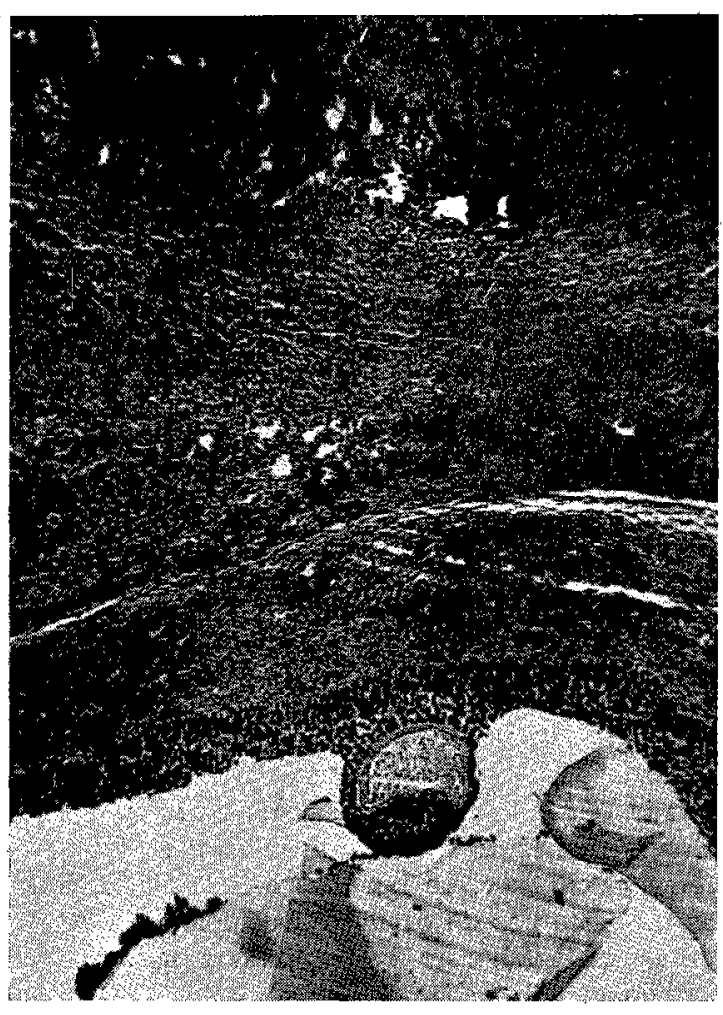

Fig. 1. - Jeune folicule de De Graat.

\section{La structure de l'ovaire.}

Les coupes de l'ovaire montrent que son cortex est formé de follicules de De Graaf sertis dans une couche de tissu conjonctif dérivé du stroma même de l'organe. En coupe, les follicules apparaissent arrondis ou triangulaires et sont disposés à l'entour du stroma dans une couche d'épaisseur uniforme, sur 2 à 4 millimètres de profondeur. En sürface, I'albuginée entoure le cortex; ce dernier recouvre l'organe, sauf dans la région du hile.

Le stroma de l'organe a 2 à 3 millimètres d'épaisseur. On remarque de nombreux follicules de $\mathrm{De}$ Graaf à divers stades de développement.

Les follicules les plus gros ont 17 et 15 millimètres environ dans leurs diamètres longitudinal et transversal.

De jeunes follicules prennent la place des gros et font saillie à la surface de l'ovaire sous forme de légères élévations arrondies ayant 5 millimetres de diamètre.

Les follicules de De Graaf translucides et piriformes prennent une apparence sphérique quand on les déloge de leur niche respective.

La majorité des gros follicules font saillie à la surface de l'ovaire et ne présentent pas de stigma à leur pourtour. Les follicules de De Graaf se situent au bord libre ventral de l'ovaire, le plus souvent près des pôles ou à leur niveau.

Quelques-uns se situent sur une face près du bord, mais ils s'accroissent tangentiellement vers les bords ou les pôles.

Si l'on compare l'ovaire de la chamelle avec celui des autres animaux domestiques, on remarque que le premier rappelle celui de la truie; mais les follicules sont aplatis chez la chamelle, sphériques chez la truie.

\section{Examen microscopique de l'ovaire.}

On utilisa l'inclusion à la paraffine.

Les colorants employés furent l'hématoxylinećosine, lo Van Gicson, le Mallory et l'orcéine.

L'ovaire est entouré par une couche mésothéliale. Les follicules sont faits de cellules épithéliales.

Le stroma est constitué par du lissu conjonctif et des fibres musculaires lisses. 'Il s'irradie en éventail et borde les couches corticales; le point de convergence se trouve vers le hile.

Les follicules. - Sur des ovaires jeunes, les follicules primaires d'une ou de plusieurs, couches occupent en abondance la couche corticale du bord ventral libre et les pôles de l'organe.

Les follicules de De Graaf. - Le stratum granu-

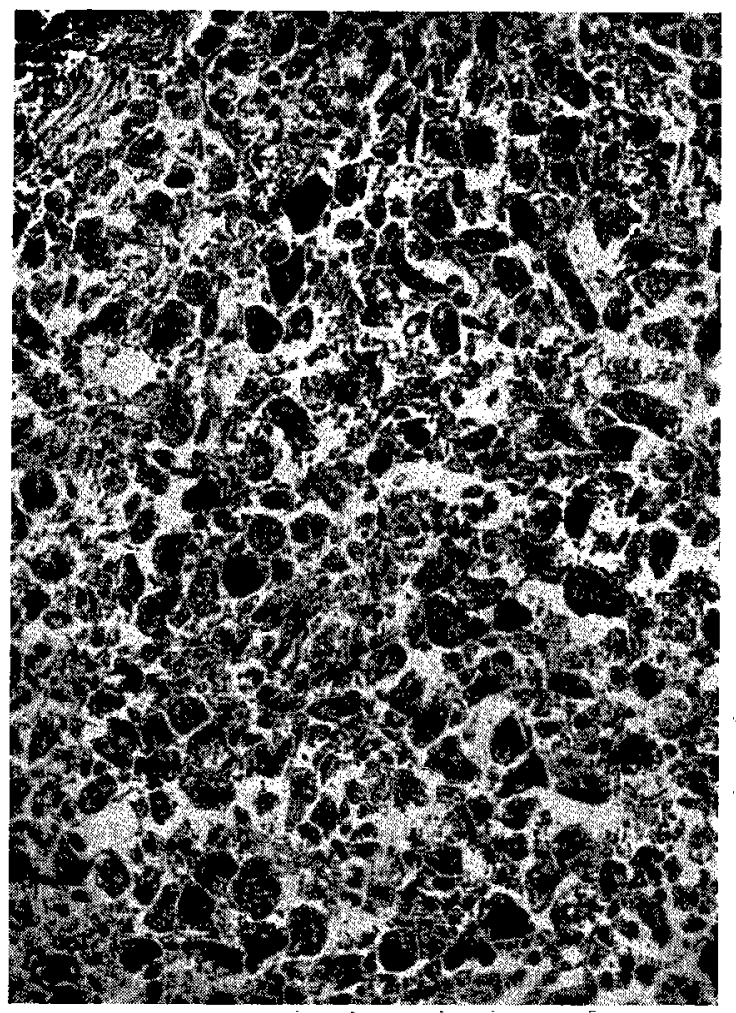

Fig. 2. C Corps jaune correspondant à un embryon de 3imillimètres. 
losum de jeunes follicules de De Graaf est formé de quatre à cinq couches de cellules, dont le noyau se colore intensément. Les cellules qui sont à proximité de la cavité folliculaire sont de forme polyédrique, à noyau rond et à cytoplasme clair non colorable. Celles qui sont au contact de la membrane basale sont en fût de colonne par leur forme avec un noyau allongé les remplissant presque totalement.

Le grand axe de ces cellules est orienté vers la cavité folliculaire. La membrane basale se met facilement en évidence. La thèque interne est faite de deux à trois couches de cellules rondes ou ovalaires avec des noyaux moins colorables et un cytoplasme légèrement teinté.

(D'ordinaire, la troisieme couche n'est pas bien définie à ce stade.) Il faut noter la présence de capillaires.

La thèque externe est très épaisse. Une couche musculaire lisse bien apparente circonscrit le follicule.

Follicules de moyenne dimension (dépassant la surface ovarienne de 1 centimètre). - Le cytoplasme des. cellules du stratum granulosum est clairement coloré. Les noyaux apparaissent vésiculeux et arrondis. Il existe six à sept couches de cellules. La forme en fût de colonne des cellules au

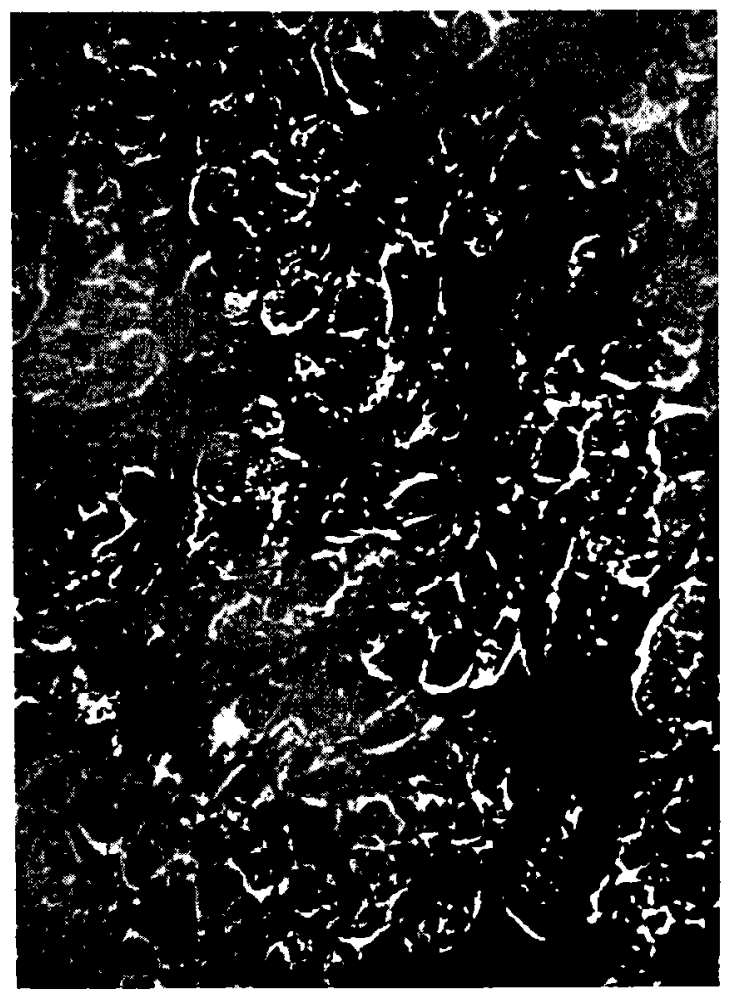

Fig. 4. - Corps jaune régressif (trous jours après la mise bas).

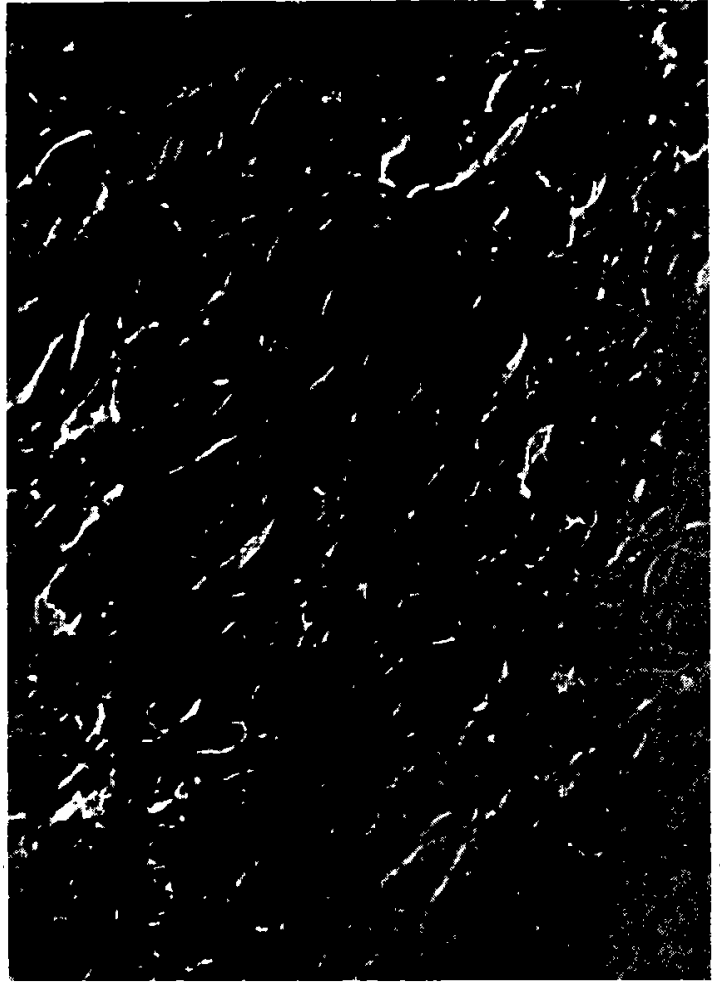

Fig. 3. - Corps jaune correspondant à un embryon de 3 centimètres. Observez les spherules $X X$ et les noyaux $N$.

contact de la membrane basalc cst conservée: La thèque interne présente maintenant trois couches de cellules épithélioïdes, de nombreux vaisseaux sanguins existent au voisinage de la membrane basale.

Follicules de 17 millimètres. - Le stratum granulosum se compose de douze a quatorze couches de cellules presque sphériques et finement granuleuses. Les noyaux sont vésiculeux et arrondis. La theque interne est faite de quatre couches de cellules épithélioides, plus grandes que celles du stratum granulosium avec de gros noyaux arrondis et vésiculeux. Une couche vasculaire est visible entre la granulosa et la thèque interne.

\section{LE CORPS JAUNE DE LA CHAMIELLE}

\section{Pendant la gestation.}

Le corps jaune se situe au bord libre ventral de l'ovaire et fait saillie hors de l'organe. Seule' une faible zone etroite se détachant du stroma ovarien reste en continuité avec la capsule thécale.

Le corps jaune apparaît de forme sphérique et de consistance molle.

Sur quelques sujets, il est allongé ou ovale. 
La capsule se compose de l'albuginée de l'ovaire et de la couche thécale sous-jacente qui conserve son apparence membraneuse jusqu'au stade où le fœtus mesure 20 centimetres.

A un stade ultérieur; la couche thécale prend une couleur blanc opaque cachant ainsi la couleur de l'amas lutéinique, de sorte que le corps jaune intégral apparait gris. gris bleuté ou même gris blanchâtre.

L'albuginée adhère fortement à la couche thécale sous-jacente et entoure presque entierement le corps jaune, ne se séparanl de ce dernier qu'au point où il se rattache à l'ovaire par un pédicule.

Des coupes longitudinales et transversales du corps jaune font apparaître des contours bien délimités, circulaires et ovalaires. La masse coagulée de liqueur folliculaire forme des lobules coniques allongés, dont le sommet se rattache au centre athrétique du corps jaune et dont la base est bien visible dans un corps jaune jeune, sous forme d'élévations arrondies à travers la capsule transparente.

Dans un corps jaune jeune la cheville centrale se réunit à une partie inversée de la capsule, de forme conique, avec un sommet dirigé vers le centre du corps jaune et remplie de caillots de sang, sauf au niveau d'une zone linéaire près de la surface qui sera intacte.

Sur des corps jaunes plus âgés la cheville centrale apparait sous forme d'une excavation de coloration blanc bleuté. Elle persiste pendant toute la gestation.

La couleur du tissu lutéinique varie au cours de la gestation; le plus jeune corps jaune rencontré correspondait à un embryon de 3 millimètres et était de couleur rouge orangé. Le corps jaune d'un fœtus de $3 \mathrm{~cm}$. 3 virait au brun orangé. Chez un foetus de 20 à 40 centimètres, la couleur du corps jaune était rouge orangé foncé.

A des stades intermédiaires et ultérieurs de la gestation la couleur s'atténue; le corps devient plus clair, et ainsi jusqu'au terme.

Le plus jeune corps jaune recueilli mesurait 18 millimètres et 15 millimètres, respectivement dans ses diamètres longitudinal et transversal.

Les dimensions du corps jaune correspondant au foetus de $3 \mathrm{~cm} .3$ étaient de 20 millimètres et 17 millimètres respectivement. Les chiffres moyens obtenus sont de 22 et 16 millimètres dans les deux dimensions ; le corps jaune le plus développé faisait 30 et 20 millimètres dans ses deux diamètres. Le poids moyen du corps jaune jusqu'au terme est de 4 grammes; le plus lourd pesait 7,5 grammes: Cependant, la taille, la forme et la position du corps jaune pendant la gestation ne varient pas jusqu'au terme.

S'il existe deux corps jaunes, la taille de chacun est inférieure à celle dı corps jaune simple correspondant.
Des vaisseaux sanguins bien développés donnent de nombreuses ramifications à la surface de la couche thécale. L'ovaire est déformé quand le corps jaune se rattache à l'une de ses faces; dans ce cas, il y a déplacement de l'organe dont le bord libre ventral se relève.

Quand le corpsjaune se rattache au pôle de l'ovaire, le grand axe de celui-ci change alors sa direction. Dans $20 \%$ des cas, il existe deux corps jaunes sur le même ovaire. Dans $3 \%$ des cas, les deux corps jaunes sont de même dimension et rattachés chacun à son ovaire. Dans un cas, trois corps jaunes se trouvaient sur le même ovaire.

Leurs diamètres étaient les suivants :

Diamètre longitudinal : 20,20 et 15 millimètres.

Diamètre transversal : 15,13 et 13 millimètres.

Dans un autre aas, l'ovaire droit comprenait deux corps jaunes mesurant 17 et 15 millimètres dans leur diamètre longitudinal et 10 millimètres chacun dans leur diamètre transversal; l'ovaire gauche possédait un corps jaune de 19 et 15 millimètres dans ses deux diamètres.

Il n'y avait qu'un seul foetus, même en présence de deux ou trois corps jaunes.

Dans un autre cas, deux foetus existaient dans la corne gauche et deux corps jaunes se trouvaient rattachés à l'ovaire correspondant.

Les deux fœus étaient de même dimension, mesuraient $3 \mathrm{~cm} .3$ de long chacun et chaque corps jaune mesurait 20 et 17 millimètres dans les deux diamètres. La maturation et la rupture de deux corps jaunes en même temps ne semblent donc pas extraordinaires chez la chamelle.

La présence de deux corps jaunes pour un seul foetus indique la perte prématurée de l'autre ovule ou un arrêt du développement embryonnaire ultérieurement.

L'ovaire gauche est loujours plus actif que le droit dans la production des ovules. Les gros follicules ne sont remarqués que dans les'premiers temps de la gestation seulement.

\section{RÉGRESSION DU CORPS JAUNE}

\section{Caractère macroscopique.}

La morphologie du corps jaune en régression a été étudiée dans quatre-vingt-dix cas (dans cinq cas on connait toute leur histoire). Le reste a été étudié d'après la taille. Les trois types suivants ont été obtenus après un accouchement normal.

1. L'animal fut abattu quatre jours après la mise bas. Le corps jaune était ferme, différent du corps jaune gestatif, ne traversait pas sa capsule, ni ne la faisait se rétracter à la section; sa couleur était brun clair; sa cavité centrale se présentait comme bien délimitée, de coloration blanc bleuté, de forme 
sphérique. Ses deux diamètres étaient respectivement de 20 et 18 millimètres.

2. L'animal fut abattu huit jours après la mise bas ; le corps jaune était dur et mesurait 21 et 17 millimètres dans ses deux dimensions. A la coupe, il apparaissait de coloration brune. La cavité centrale était nette.

3. L'animal allaitait son petit depuis trente jours et fut abattu le trente et unième. Il y avait deux corps jaunes; l'un était blanc grisâtre, dur, de forme sphérique, de dimensions 19 et 15 millimètres. A la section, la couche capsulaire était épaisse de 1 millimètre et la cavité centrale était bien définie. Le second était gris noirâtre, tendu, de forme ovalaire. A la section, une matière brun noirâtre fit irruption; le reste ressemblait à une tête pédonculée, contenant du poil.

L'examen microscopique démontra qu'il s'agissait d'un dermoïde. Un autre cas fut signalé, mais on ne connaissait pas les commémoratifs.

Les exemples suivants sont tirés de l'étude d'animaux ayant avorté.

4. L'animal fut abattu vingt-quatre heures après. Le corps jaune rappelait le corps jaune gestatif et mesurait 17 et 13 millimètres dans ses deux diamètres.

3. L'animal fut abattu au bout de quatre jours. Le corps jaune était légèrement plus ferme que dans le premier exemple. A la section, la coloration était foncée également; l'ovaire et les follicules de De Graaf étaient rouge foncé. D'après les exemples cités et d'après ceux pour lesquels les commémoratifs sont incertains, il apparaît que le corps jaune de la chamelle change de consistance (de mou il devient dur) une semaine environ après la naissance ou l'avortement.

Bien que le corps jaune se réduise progressivement quant à ses dimensions, il fait saillie encore longtemps sur l'une des faces de l'ovaire ou à son bord libre et se présente comme une sphère grisâtre comprimée latéralement. Les corps jaunes les plus âgés sont blanc grisâtre ou blanc jaunâtre, durs, ont l'aspect d'élévations en bouton aux points d'insertion habituels, de dimensions égales (5 millimètres dans les deux diamètres), le plus souvent enfouis dans le cortex.

On remarque que le corps jaune régressif décroît d'abord dans son diamètre transversal, puis dans son diamètre longitudinal, enfin d'une manière alternée. Sur une coupe transversale, les corps jaunes de moins de 14 millimètres de longueur varient de la couleur brun à la couleur chocolat. Quand les corps jaunes ont 7 millimètres de long, leur cavité centrale commence à disparaitre. La coloration blanche du corpus albicans n'est vue que sur les corps jaunes de 5 millimètres. La capsule thécale atteint, dans quelques cas, 3 millimètres d'épaisseur environ.

\section{Caractères microscopiques.}

L'inclusion à la paraffine, la congélation et la coupe au microtome (sur des pièces frâ̂ches) furent utilisées.

Les colorants employés (sur des pièces fraîches) furent l'hématoxyline-éosine, le Van Gieson, le Mallory et l'acide osmique.

Dans le corps jaune se rapportant à l'embryon de 3 millimètres, les cellules lutéiniques étaient grandes, avec un gros noyau arrondi. Le contour de ces cellules prenait mal les colorants et apparaissait assez flou, le cytoplasme était semé de vacuoles.

D'autres vacuoles, plus grandes ceiles-ci, contenaient des sphérules, de même couleur que le cytoplasine, mais en différant par leur indice de réfraction (sur des pièces fraîches les cellules étaient ovales).

Les cellules lutéiniques n'étaient ! pas soutenues directement par les fibres conjonctives, mais cellesci entouraient des groupes de ces mêmes cellules un peu comme si elles s'étaient trouvées dans une cuvette.

Ces groupes cellulaires n'avaient pas une disposition en rangées bien définies, comme on peut le remarquer sur des corps jaunes plus âgés. Le cytoplasme prenait bien les colorants rouge, brun et orange (respectivement l'hématoxyline-éosine, le van Gieson et le Mallory).

Sur le corps jaune se rapportant au foetus de 3 centimètres, les cellules devenaient plus grandes avec un contour moins net; elles se présentaient sous forme d'oves allongées et étaient disposées en rangées bien déterminées séparées par des cloisons de tissu conjonctif. Les espaces compris entre les cloisons étaient triangulaires à la section. Le sommet regardait vers le centre du corps jaune.

Les sphérules signalées plus haut prenaient de l'ampleur. Le noyau occupait diverses positions dans la cellule.

Sur le corps jaune se rapportant à un fœtus de 61 centimètres les cellules prenaient une apparence spumeuse avec une membrane nette et de forme ovalaire et un noyau faiblement coloré. I,es trabécules de tissu conjonctif augmentaient de nombre.

Le corps jaune régressif. - Trois jours après la naissance, la capsule s'épaissit et chaque cellulo lutéinique est entourée de fibres conjonctives.

Trente jours après la mise bas, les cellules lutéiniques sont le plus souvent dégénérées; les noyaux forment des agrégats en taches au sein du tissu conjonctif.

Sur des corps jaunes plus âgés, la thèque externe est devenue du tissu conjonctif fibreux dense.

\section{POSITION DU FETUS DANS L'UTÉRUS}

Tous les fœetus examinés occupaient la corne gauche et partiellement le corps de l'utérus. On n'en vit jamais dans la corne droite. 
L'examen de plusieurs utérus vides à divers stades d'involution a montré que la corne gauche était plus volumineuse que la corne droite.

La gestation à gauche semble donc être habituelle chez la chamelle.

Le foetus lo plus long observé mesurait 110 centimètres.

Le plus petit nouveau-né mesurait 104 centimètres et le plus long 122 centinèlres.

Sur 20 nouveau-nés, la moyenne des longueurs a été de 116 centimètres.

D'après LEESE (1927), la durée de gestation est de douze mois et selon ASDELL (1946), quatre centsix jours.

\section{RÉSUMÉ}

Cent cinquante ovaires ont été étudiés in situ chez la chamelle soudanaise aux abattoirs du Caire et d'Embaba.

Ijablation en fut ensuite pratiquée pour l'examen des corps jaunes. Soixante ovaires provenaient de femelles pleines et quatre-vingt-dix de femelles non gestantes. Ces 'derniers portaient des corps jaunes à divers stades de régression.

Los rósultats de cos oxamcns sont los suivants : $1^{0}$ Les ovaires de la chamelle ne présentent pas de cavité d'ovulation et sont situés dans leur bourse ovarique

$2^{\circ}$ Il n'y a pas d'attache entre le morceau frangé et l'ovaire.

$3^{\circ}$ Les follicules de De Graaf sont des spheres claires et translucides, sans stigma apparent, faisant saillie à la surface de l'ovaire; ils sont situés au bord ventral libre de l'ovaire, ordinairement à proximité des pôles.

$4^{\circ} \mathrm{L}$ 'examen microscopique de ces ovaires révèle les mêmes caracteristiques que pour ceux des autres animaux domestiques. $5^{\circ} \mathrm{Au}$ cours de la gestation, le corps jaune apparaît comme une sphère molle, comprimée latéralement, faisant saillie sur l'ovaire; sa forme, son, poids, sa taille ne varient pas; seule sa couleur change.

$6^{\circ}$ On trouve fréquemment une paire de corps jaunes sur le même ovaire avec un seul fœetus dans l'utérus.

$7^{\circ} \mathrm{L}$ 'ovaire gauche a une production ovulaire plus intense que l'ovaire druit. La gestalion dans la corne gauche est la plus fréquente.

$8^{\circ}$ L'examen microscopique des corps jaunes au cours de la gestation révele une diminution des lipoïdes et des graisses dans les cellules lutéiniques, ainsi qu'un accroissement di tissu conjonctif, au fur et à mesure quion approche du part.

$9^{\circ}$ Le corps jaune se transforme en une sphère dure et comprimée latéralement, une semaine environ après la naissance ou l'avortement, puis décroît ensuite progressivement d'abord transversalement, puis longitudinalement, ensuite d'une façon alternée.

$10^{\circ}$ La dégénérescence des cellules lutéiniques dans le corps jaune en régression semble due à l'épaississement progressif de la capsule thécale et à l'invasion de tissu conjonctif autour de chaque cellule, rompant ainsi son irrigation sanguine.

$11^{\circ}$ La dégénérescence dermoîde du cor̀ps jaune est possible.

\section{BIBLIOGRAPHIE}

1. LESBRE (M,-F, $\left.X_{1}\right)$, - Recherches anatomiques sur les Camélidés. Arch. Mus. Nat. VIII, 1903.

2. LEESE (A.-S.). '- $\mathbf{A}$ treatise on the one-humpel camel, published by Haynes and Sons. Maiden Larie. Stamford, Lincolnshire, 1927.

3. ASDELL (A.-S.). - Patterns of the mammalian reproduction, Ithaca, New-York, Comstock Publishing Co. Inc., 1946

4. HAMMOND (J.). - The physiology of the reproduction of the cow, Cambridge University Press, Fetter Lane', London, 1927. 